OPEN ACCESS

Edited by:

Laura Maggi,

Università degli Studi di Firenze, Italy

Reviewed by:

Jonilson Berlink Lima,

Federal University of Western Bahia,

Brazil

Agnieszka Rusak,

Wroclaw Medical University, Poland

${ }^{*}$ Correspondence:

Huang Fang

fanghuangtih@126.com

Huan Wang

wanghuan@tih.tjmu.edu.cn

Specialty section: This article was submitted to

Cytokines and Soluble

Mediators in Immunity,

a section of the journal

Frontiers in Immunology

Received: 05 March 2021

Accepted: 24 May 2021

Published: 08 June 2021

Citation:

Li L, Wei K, Ding Y, Ahati $P, X u H$,

Fang $\mathrm{H}$ and Wang $\mathrm{H}$ (2021) M2a

Macrophage-Secreted CHI3L1

Promotes Extracellular Matrix

Metabolic Imbalances via Activation of

IL-13Ro2/MAPK Pathway in Rat Intervertebral Disc Degeneration.

Front. Immunol. 12:666361.

doi: 10.3389/fimmu.2021.666361

\section{M2a Macrophage-Secreted CHI3L1 Promotes Extracellular Matrix Metabolic Imbalances via Activation of IL-13R $\alpha 2 / M A P K$ Pathway in Rat Intervertebral Disc Degeneration}

\author{
Long Li, Kang Wei, Yifan Ding, Paerxiati Ahati, Haoran Xu, Huang Fang * and Huan Wang \\ Department of Orthopedics, Tongji Hospital, Tongji Medical College, Huazhong University of Science and Technology, \\ Wuhan, China
}

The accumulation of macrophages in degenerated discs is a common phenomenon. However, the roles and mechanisms of $\mathrm{M} 2 \mathrm{a}$ macrophages in intervertebral disc degeneration (IDD) have not been illuminated. This study investigated the expression of the M2a macrophage marker (CD206) in human and rat intervertebral disc tissues by immunohistochemistry. To explore the roles of M2a macrophages in IDD, nucleus pulposus (NP) cells were co-cultured with M2a macrophages in vitro. To clarify whether the $\mathrm{CHI}$ L 1 protein mediates the effect of M2a macrophages on NP cells, siRNA was used to knock down $\mathrm{CHI}$ L1 1 transcription. To elucidate the underlying mechanisms, NP cells were incubated with recombinant $\mathrm{CH} 3 \mathrm{~L} \mathrm{~L} 1$ proteins, then subjected to western blotting analysis of the IL-13R 2 2 receptor and MAPK pathway. CD206-positive cells were detected in degenerated human and rat intervertebral disc tissues. Notably, M2a macrophages promoted the expression of catabolism genes (MMP-3 and MMP-9) and suppressed the expression of anabolism genes (aggrecan and collagen II) in NP cells. These effects were abrogated by $\mathrm{CH} 3 \mathrm{~L} 1$ knockdown in M2a macrophages. Exposure to recombinant $\mathrm{CHI}$ L1 promoted an extracellular matrix metabolic imbalance in NP cells via the IL-13R $\alpha 2$ receptor, along with activation of the ERK and JNK MAPK signaling pathways. This study elucidated the roles of M2a macrophages in IDD and identified potential mechanisms for these effects.

Keywords: intervertebral disc degeneration, M2a macrophage, nucleus pulposus cell, CHI3L1, MAPK 


\section{INTRODUCTION}

Low back pain has been an quite common disorder (1), experienced by more than 500 million people worldwide. Lower back pain is the first leading cause of disability among individuals of working age. Intervertebral disc degeneration (IDD) (2), an age-related chronic pathological process, is generally regarded as a primary cause of this disease $(3,4)$.

An imbalance between the synthesis and degradation of extracellular matrix (ECM) secreted by NP cells has been identified as the main pathophysiological process of IDD (5). In addition, multiple studies have demonstrated the accumulation of macrophages in degenerated intervertebral disc tissues $(6,7)$. However, the potential roles of macrophages in the onset of IDD have not been fully elucidated $(8,9)$, partly because of substantial heterogeneity among macrophages. Understanding these roles is critical for developing new IDD treatments based on the underlying pathophysiology (10).

Macrophages exhibit multiple phenotypes (11), commonly divided into M1, M2a, and M2c subsets (12), identified by cell surface markers CCR7, CD206, and CD163 (13), respectively. Recently, Nakazawa et al. reported that all three types of macrophage markers were present in degenerated intervertebral disc tissues (14). Although several studies have tried to investigate the roles of macrophages in IDD (9), there has been minimal consideration of specific types of macrophage, especially M2a (CD206) macrophages (8).

In M2a macrophages, the expression of chitinase 3-like 1 protein (CHI3L1) is elevated, compared with other macrophage types (15). CHI3L1 is a secreted glycoprotein that can facilitate tumor invasion and metastasis by upregulating the expression levels of matrix metalloproteinase (MMP) genes in various tumor cells (16-18). In many inflammatory diseases (e.g., rheumatoid arthritis and liver cirrhosis), CHI3L1 is strongly expressed by macrophages and can mediate proinflammatory effects $(19,20)$. Thus, we hypothesized that M2a macrophages secrete $\mathrm{CHI} 3 \mathrm{~L} 1$, thus contributing to the pathological progression of IDD.

To test our hypothesis, we investigated the infiltration of M2a macrophages in human and rat degenerated NP tissues by means of immunohistochemistry. Subsequently, we induced M2a polarization from human monocyte THP-1 cells by stimulation with interleukin (IL)-4, which is the strongest inducer of the M2a phenotype (21). By using a co-culture system, we found that M2a macrophages exacerbated a metabolic imbalance in the ECM, partly through secretion of CHI3L1 protein. Finally, we found that IL-13R $\alpha 2$ and the mitogen-activated protein kinase (MAPK) signaling pathway were involved in this process.

\section{MATERIALS AND METHODS}

\section{Clinical Sample Collection and Ethical Considerations}

The study was approved by the Ethics Committee of Tongji Hospital, Tongji Medical College, Huazhong University of

Abbreviations: IDD, Intervertebral disc degeneration; NP, Nucleus pulposus; ECM, Extracellular matrix; MAPK, Mitogen-activated protein kinase.
Science and Technology. All patients signed informed consent forms. In total, 18 human intervertebral disc NP tissues were collected from surgeries in which intervertebral disc resection was required. Among the samples, 10 were obtained from patients with IDD (IDD group, seven female patients and three male patients; mean age $=52 \pm 8$ years) and eight were obtained from patients with adolescent idiopathic scoliosis or congenital scoliosis without signs of disc degeneration (22) (normal group, five female patients and three male patients; mean age $=15 \pm$ 6 years).

\section{Animal Model}

The animal study was reviewed and approved by Ethics Committee of Tongji Hospital, Tongji Medical College, Huazhong University of Science and Technology. SpragueDawley rats were purchased from the Hubei Province Experimental Animal Center (Wuhan, China). A rat IDD model was established in the coccygeal vertebra through needle puncture, as described previously (23). Briefly, 12 SpragueDawley rats (6-8 weeks old, female) were randomly divided into normal and IDD model groups ( $\mathrm{n}=6$ rats per group). After induction of anesthesia, discs corresponding to Co $8 / 9$ caudal vertebral gaps were identified as puncture points. A $20-G$ puncture needle was inserted into the rat tail until complete penetration was achieved, rotated $360^{\circ}$ and maintained for $30 \mathrm{~s}$, and then removed. Two months after surgery, magnetic resonance imaging was performed to confirm that degeneration modeling had been successful.

\section{Sample Processing and Immunohistochemistry}

Rat NP tissues and a small portion of adjacent vertebrae were harvested 2 months after surgery. After tissues had been fixed for $24 \mathrm{~h}$ in $10 \%$ formaldehyde, they were soaked in ethylenediaminetetraacetic acid decalcifying solution for 2 months and embedded in paraffin. Human NP tissues were fixed in $4 \%$ paraformaldehyde for $2 \mathrm{~h}$ before paraffin embedding. All paraffin-embedded tissues were cutted into 5$\mu \mathrm{m}$ sections for subsequent immunohistochemical analysis.

Immunohistochemical staining was performed to examine CD206 expression in human or rat NP tissues, as described previously (24). Briefly, slices were deparaffinized for $15 \mathrm{~min}$ in xylene and dehydrated in a graded ethanol series (from $99 \%$ to 75\%) for 20 min per solution. For antigen retrieval, sections were incubated in $0.01 \mathrm{M}$ citrate buffer $(\mathrm{pH} 6.0)$ for $20 \mathrm{~min}$ at $95^{\circ} \mathrm{C}$. Endogenous peroxidases were inhibited by incubation in $3 \%$ $\mathrm{H} 2 \mathrm{O} 2$ at $37^{\circ} \mathrm{C}$ for $10 \mathrm{~min}$. After blocking with goat serum for $30 \mathrm{~min}$ at room temperature, primary anti-CD206 antibody (ab64693, Abcam, Cambridge, UK) was incubated overnight at $4^{\circ} \mathrm{C}$. After incubation with horseradish peroxidase-conjugated goat anti-rabbit IgG for $1 \mathrm{~h}$ at room temperature, cellular nuclei staining was performed by diaminobenzidine treatment and counterstaining with hematoxylin for $3 \mathrm{~min}$. In between all incubation steps, sections were washed three times with phosphate-buffered saline (PBS; 5 min per wash). Finally, slices were photographed under a light microscope. The ratio of 
positive cells and the mean optical density were analyzed with Image software, version 2.0.

\section{Rat NP Cells Extraction and Treatment}

Rat NP cells were isolated from the coccygeal intervertebral discs of Sprague-Dawley rats (6-8 week old) as described before (25). Briefly, gelatinous NP tissues were isolated under aseptic conditions and then digested in $0.25 \%$ trypsin for $30 \mathrm{~min}$ and $0.2 \%$ type II collagenase for $3 \mathrm{~h}$ at $37^{\circ} \mathrm{C}$. After sufficient digestion, the tissues were suspended in DMEM/F12 medium (HyClone, Logan, UT, USA) containing 10\% fetal bovine serum (HyClone) and $1 \%$ streptomycin/penicillin, then incubated at $37^{\circ} \mathrm{C}$ in a humidified $5 \% \mathrm{CO}_{2}$ incubator. Passages were conducted after cell fusion and the first three passages of cells were used for subsequent experiments.

\section{M2a Macrophage Polarization and Co-Culture}

Human THP-1 monocytes were differentiated into M0 or M2a macrophages, as described previously $(26,27)$. Briefly, human monocyte cell line THP-1 cells were stimulated with $100 \mathrm{ng} / \mathrm{ml}$ phorbol 12-myristate 13-acetate (PMA, P1585, Sigma, USA) for $12 \mathrm{~h}$ to achieve M0 polarization. Subsequently, M0 macrophages were incubated continually with $20 \mathrm{ng} / \mathrm{ml}$ of human recombinant IL-4 (200-04, PeproTech, USA) for $24 \mathrm{~h}$ to generate M2a macrophages. Using a Transwell co-culture system $(0.4-\mu \mathrm{m}$ pore size, Costar, Corning, NY, USA), M2a macrophages and NP cells were co-cultured for $48 \mathrm{~h}$.

\section{Immunofluorescence}

After fixing with $4 \%$ paraformaldehyde for 15 minutes, treated cells were permeabilized and blocked with $5 \%$ bovine serum albumin/0.3\% Triton ${ }^{\text {TM }}$ X-100 PBS for $1 \mathrm{~h}$ at room temperature. Primary antibodies against CD206 (ab64693, Abcam), CHI3L1 (ab180569, Abcam), or IL-13R $\alpha 2$ (ab108534, Abcam) were applied and incubated overnight at $4^{\circ} \mathrm{C}$. Secondary antibody were incubated with Cy3-conjugated goat anti-rabbit IgG (1:100, BA1032, Boster, Wuhan, China) or fluorescein isothiocyanateconjugated goat anti-mouse IgG (1:100, BA1101, Boster) for $1 \mathrm{~h}$. For nuclear counterstaining, DAPI was incubated for $10 \mathrm{~min}$. Cells were protected from light during incubation with secondary antibodies and DAPI. In between all incubation steps, cells were washed three times with PBS ( $5 \mathrm{~min}$ per wash). Cells were examined and photographed by fluorescence microscopy.

\section{siRNA Knockdown Experiments}

Knockdown of the CHI3L1 gene in M2a macrophages or the IL$13 \mathrm{R} \alpha 2$ gene in NP cells was achieved by transfection with siRNA (targeting CHI3L1 or IL-13R $\alpha 2$, or a scrambled sequence as negative control), using Lipofectamine 2000 (Invitrogen, USA) (28). siRNA sequences were as follows: CHI3L1, 5'-CCAUAUCA UCUACAGCUUUTT-3' and 5'-AAAGCUGUAGAUGA UAUGGGT-3'; IL-13R 22, 5'-GGGUUAUCUCUAUUUG CAATT-3' and 5' UUGCAAAUAGAGAUAACCCAG-3'. Knockdown efficacy was detected by western blotting.

\section{Western Blotting Analysis}

Cellular proteins $(25 \mu \mathrm{g})$ were separated and probed with primary antibodies to MMP3 (17873-1-AP, Proteintech), MMP9 (10375-2-AP, Proteintech), COL2A1 (15943-1-AP, Proteintech), aggrecan (13880-1-AP, Proteintech), phosphoJNK (\#4668, CST), JNK (\#9252, CST), phospho-ERK (\#4370, CST), ERK (\#4695, CST), phospho-p38 (\#4511, CST), p38 (\#8690, CST), and GAPDH (60004-1-Ig, Proteintech). Following incubation with primary antibody overnight at $4^{\circ} \mathrm{C}$, HRP-conjugated anti-rabbit or anti-mouse secondary antibodies (BA1054, BA1050, Boster) were incubated; protein bands were visualized using an enhanced chemiluminescence system (Thermo Fisher Scientific) (29). Quantification of western blotting results was performed using ImageJ software, version 2.0 .

\section{Quantitative Reverse-Transcription PCR (qRT-PCR)}

Total RNA was isolated with TRIzol reagent (Invitrogen) and cDNA was reverse transcribed using the Reverse Transcription Kit (Toyobo, Japan). The qRT-PCR experiments were performed on a Bio-Rad iQ5 real-time PCR detection system using SYBR Green reagent (Toyobo) (30). Primer sequences are listed in Table 1.

\section{Statistical Analysis}

GraphPad Prism 5 software (GraphPad Inc., La Jolla, CA, USA) was used for statistical analysis. All quantitative results are shown as means \pm standard deviations. Data between two groups were compared using the Student's t -test. One-way analysis of variance (ANOVA) was used to compare data among three or more groups. Percentages were compared by Fisher's exact test using R software, version 3.5.3. A p value $<0.01$ was regarded as statistically significant.

\section{RESULTS}

\section{Expression of the M2a Macrophage Marker, CD206, in Human and Rat Degenerated Intervertebral Disc Tissues}

To evaluate the accumulation of M2a macrophages in degenerative intervertebral disc tissues, immunohistochemical analysis of CD206 (a surface marker for M2a) was performed

TABLE 1 | Primers used in this study.

\begin{tabular}{lcll}
\hline Gene & Species & \multicolumn{1}{c}{ Forward } & \multicolumn{1}{c}{ Reverse } \\
\hline CD206 & Human & aacggactgggttgctatca & cccgatcccttgtagagcat \\
CD301 & Human & gctgctggtcatcatctgtg & cctccacctcagctttcaga \\
Arginase1 & Human & gtggaagaaggccctacagt & gcttttcccacagaccttgg \\
GAPDH & Human & ccaaggagtaagacccctgg & tggttgagcacagggtactt \\
MMP3 & Rat & atgacagggaagctggactc & ctggagaatgtgagtggggt \\
MMP9 & Rat & aggatggtctactggcacac & gtgcaggacaaataggagcg \\
COL2A1 & Rat & tgttgacattgcacccatgg & cagccattcagtgcagatcc \\
Aggrecan & Rat & catgcatcctgtgaccactg & gcatcacttcacagcggtag \\
GAPDH & Rat & agacagccgcatcttcttgt & cttgccgtgggtagagtcat
\end{tabular}


using human and rat intervertebral disc tissues. Analysis of human intervertebral disc tissues showed that CD206-positive cells were absent from normal disc samples (0/8, 0\%), although seven of the 10 IDD samples exhibited at least one CD206positive cell $(7 / 10,70 \%)$ (Figures 1A, B). Analysis of rat intervertebral disc tissues showed significantly elevated expression of CD206 in degenerated intervertebral disc tissues, compared with the normal group (Figures 1C, D). Furthermore, CD206-positive cells in rat discs closely resembled resident NP cells, indicating that NP cells may also express marker of M2a macrophages. These findings indicated that significantly greater numbers of CD206-positive cells were present in degenerated disc tissues, comparing with normal group.

\section{Contribution of M2a Macrophages to ECM Metabolic Imbalance in NP Cells}

To explore the effects of M2a macrophages on ECM metabolism, the macrophages were co-cultured with NP cells. Firstly, THP-1 monocytes were polarized into M0 (i.e., control) and M2a macrophages. Immunofluorescence staining revealed that the expression levels of CD206 were significantly elevated in M2a macrophages, compared with the levels in M0 macrophages (Figures 2A, B). Western blotting results confirmed this difference in expression levels (Figures 2C, D). To confirm M2a macrophage polarization, the mRNA expression levels of M2a-associated genes were measured by qRT-PCR. Importantly, the expression levels of CD204, CD206, and arginase-1 were significantly upregulated in M2a macrophages (Figure 2E). After successful polarization had been confirmed, M0 and M2a macrophages were co-cultured with $\mathrm{NP}$ cells. After $48 \mathrm{~h}$ of co-culture, the expression levels of anabolism genes (aggrecan and collagen II) and catabolism genes (MMP-3 and MMP-9) were detected by qRT-PCR. The results showed enhanced transcription of anabolism genes and reduced expression of catabolism genes in the M2a co-culture group, compared with the untreated and M0 co-culture groups (Figure 2F). Thus, M2a macrophages promoted ECM metabolic imbalance in NP cells.

A
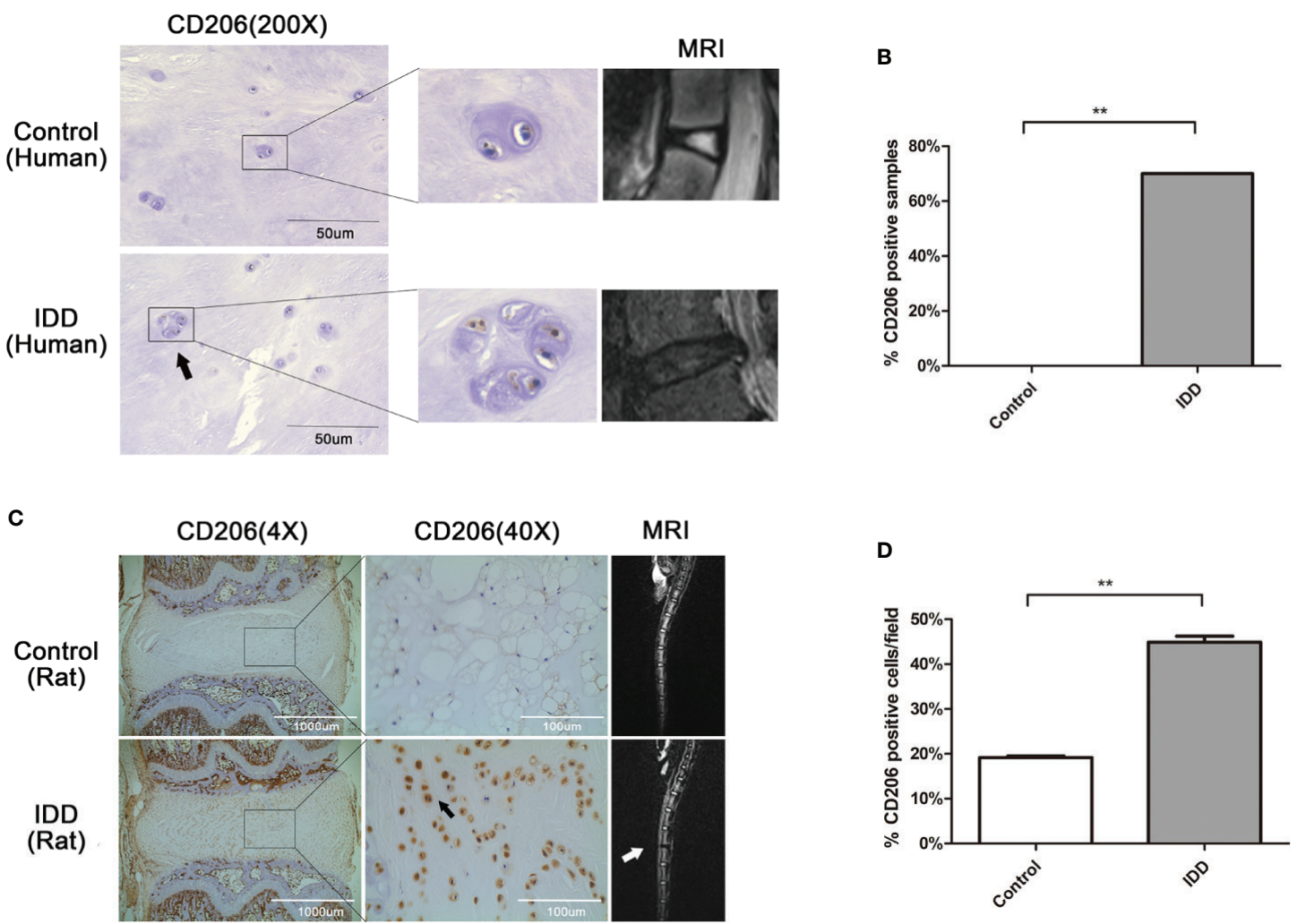

FIGURE 1 | Expression of the M2a macrophage marker, CD206, in human and rat degenerated intervertebral disc tissues. (A) Immunolocalization of CD206positive cells in human intervertebral disc tissues. CD206-positive cells were detected using immunofluorescence staining with an anti-CD206 antibody. Representative pictures are from a patient with congenital scoliosis (11-year-old girl) and a patient with IDD (56-year-old woman), representing normal and IDD samples, respectively. (B) The ratio of CD206-positive samples between normal and IDD samples. CD206-positive cells were absent from normal disc samples (0/8, 0\%), although seven of the 10 IDD samples exhibited at least one CD206-positive cell (7/10, 70\%); Fisher's exact test, ${ }^{* *} \mathrm{p}<0.01$. (C) Immunolocalization of CD206positive cells in rat intervertebral disc tissues. Intervertebral disc degeneration in a rat model was induced by needle puncture, and immunohistochemical staining of intervertebral disc tissues was performed with an anti-CD206 antibody. (D) Quantitative analysis of CD206 expression, the dates were analyzed by Student's t -test. Significant differences between groups are shown as ${ }^{* \star} p<0.01$. 

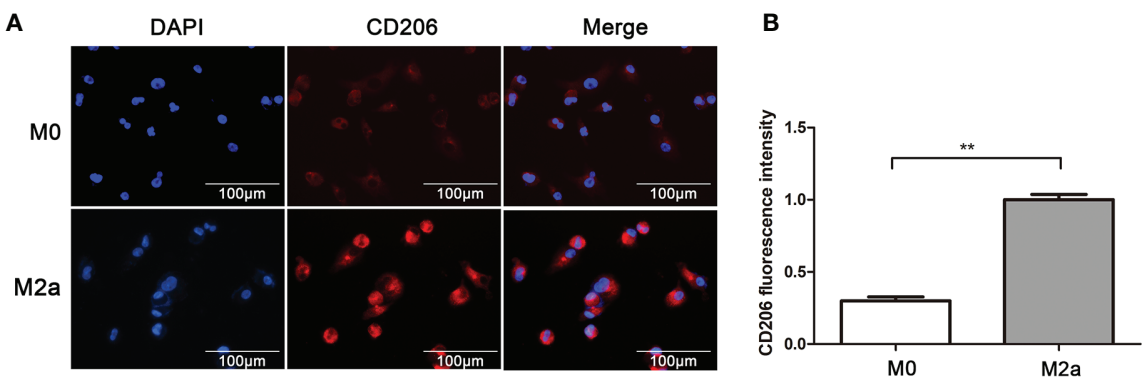

C

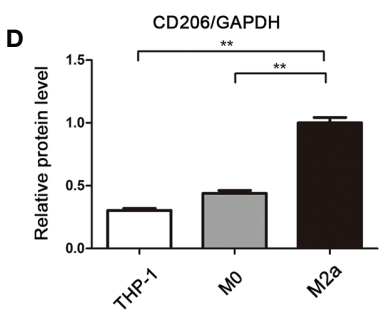

E
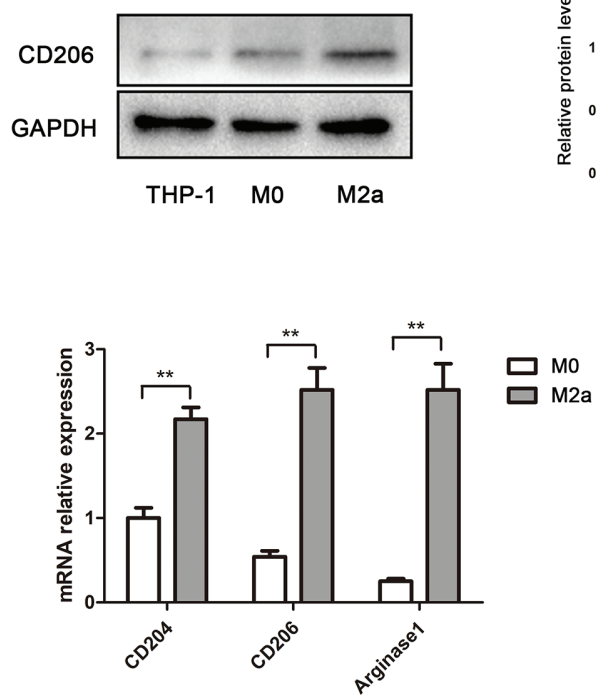

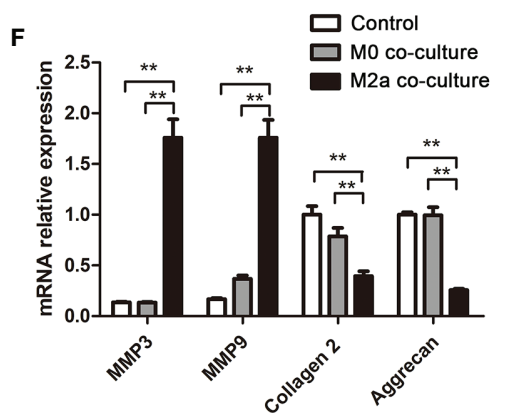

FIGURE 2 | M2a macrophages promoted ECM degradation in NP cells. (A) Immunofluorescence analysis of the CD206 differentiation marker in M2a macrophages. $\mathrm{MO}$ and M2a macrophages were differentiated from THP-1 monocytes by stimulation with phorbol 12-myristate 13-acetate or IL-4, respectively. Immunofluorescence staining for CD206 was performed. Scalebar, $100 \mu \mathrm{m}$. (B) Semi-quantitative analysis of CD206 immunofluorescence staining results. (C) Western blotting analysis of CD206 differentiation marker in M2a macrophages. M0 and M2a macrophages were differentiated from THP-1 monocytes. The protein levels of CD206 in THP-1, M0 (i.e., control), and M2a macrophages were determined by western blotting. (D) Quantification of CD206 protein levels in western blots. (E) qRT-PCR analysis of M2a macrophage differentiation marker genes (CD204, CD206, and arginase-1). (F) Effects of M2a macrophages on NP cells via Transwell co-culture. M0 and M2a macrophages were differentiated from THP-1 monocytes, then co-cultured with NP cells for 48 h. MMP3, MMP9, collagen II, and aggrecan expression levels of NP cells were analyzed by qRT-PCR. Experiments were repeated three times in triplicate. The dates were analyzed by One-way analysis of variance (ANOVA).

Statistically significant difference: ${ }^{\star \star} \mathrm{P}<0.01$.

\section{Role of CHI3L1 in Induction of ECM Metabolic Imbalance by M2a Macrophages}

To determine whether CHI3L1 mediated M2a macrophageinduced ECM metabolic imbalance in NP cells, CHI3L1 secretion was investigated in M2a macrophages. Western blotting analysis showed high expression of the CHI3L1 protein in M2a macrophages (Figures 3A, B). Subsequently, $\mathrm{CHI}$ L1 gene expression in M2a macrophages was silenced by CHI3L1-siRNA, and effective knockdown was confirmed by western blotting (Figures 3C, D). The results showed that CHI3L1 knockdown abolished the M2a macrophage-induced ECM metabolic imbalance (Figure 3E). Taken together, these findings implied that CHI3L1 mediated M2a macrophageinduced ECM metabolic imbalance.

\section{Effects of Recombinant CHI3L1 (rCHI3L1) on ECM Degradation}

To explore further the effects of CHI3L1 on ECM metabolism, rCHI3L1 was added to NP cells at different concentrations or for different intervals. Notably, rCHI3L1 significantly upregulated the expression of catabolic genes MMP3 and MMP9, and downregulated the expression of anabolism genes aggrecan and collagen II (Figure 4). These effects were concentrationand time-dependent.

\section{Role of IL-13R $\alpha 2$ in CHI3L1-Induced ECM Degradation in NP Cells}

To clarify the mechanism responsible for the effects of CHI3L1 on NP cells, a potential receptor for CHI3L1, IL-13R $\alpha 2$, was then 
A

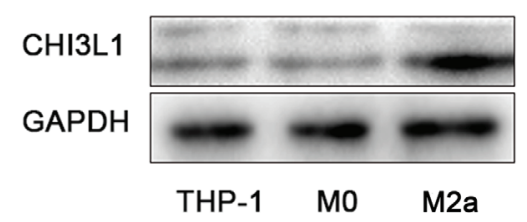

C

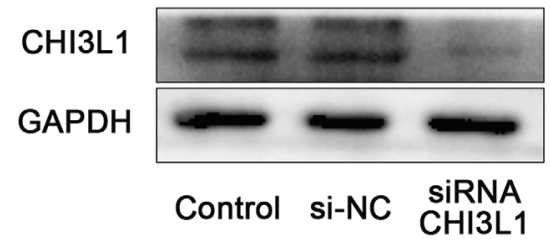

E

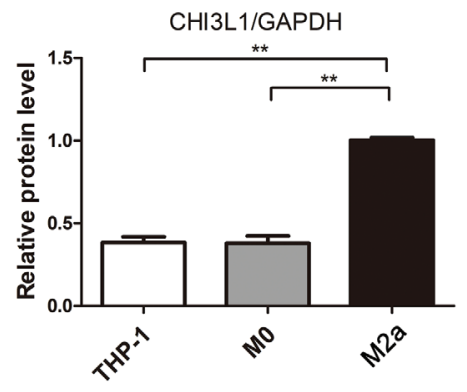

D

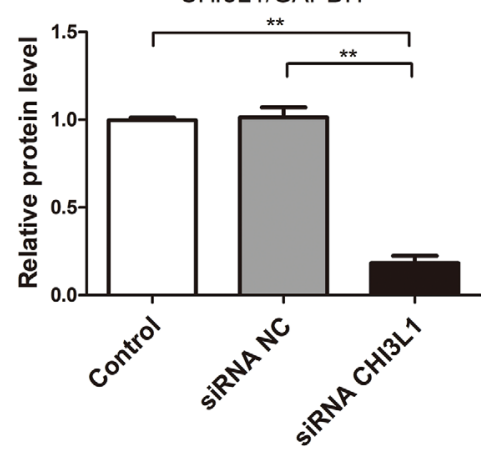

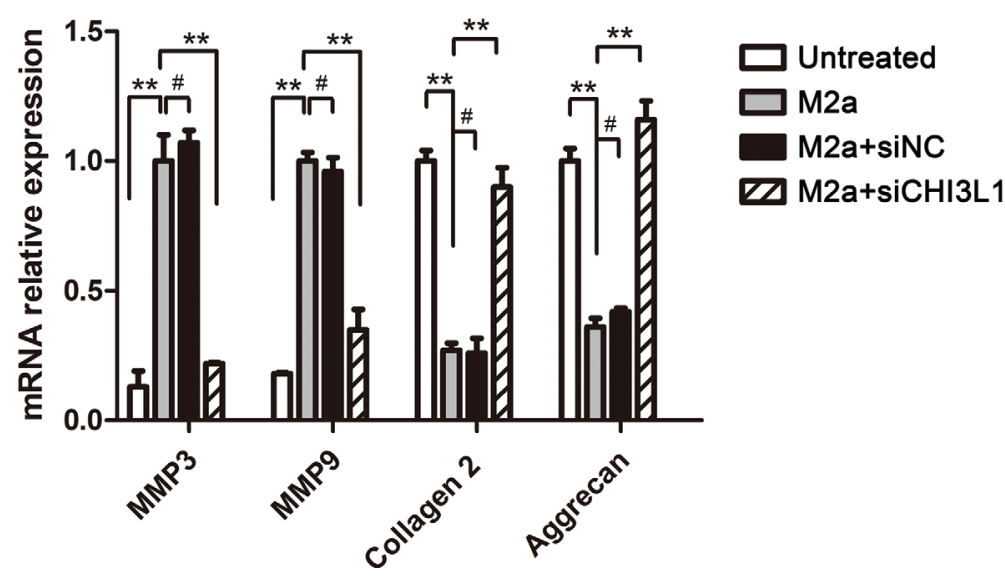

FIGURE 3 | CHI3L1 mediated M2a macrophage-induced ECM degradation. (A) Expression of CHI3L1 in M2a macrophages. M0 and M2a macrophages were differentiated from THP-1 monocytes. The protein levels of CHI3L1 in THP-1, MO and M2a macrophages were detected by western blotting. (B) Quantification of CHI3L1 protein levels in western blots. (C) siRNA silencing of CHI3L1 in M2a macrophages. After transfection with NC siRNA or CHI3L1 siRNA, the knockdown of CHI3L1 in M2a macrophages was confirmed by western blotting. (D) Quantification of CHI3L1 protein levels in western blots. (E) Effects of CHI3L1 silencing in M2a macrophages on ECM degradation in NP cells. After co-culture with CHI3L1-silenced M2a macrophages, the expression levels of MMP3, MMP9, collagen II and aggrecan in NP cells were analyzed by qRT-PCR. Experiments were repeated three times in triplicate. The dates were analyzed by One-way analysis of variance (ANOVA). Significant differences between groups are shown as ${ }^{* *} \mathrm{p}<0.01$. \#, no statistical difference.

investigated. After treatment with $\mathrm{rCHI} 3 \mathrm{~L} 1$, double immunofluorescence staining showed co-localization of CHI3L1 and IL-13R $\alpha 2$ in NP cells (Figure 5A). To explore further whether the IL-13R $\alpha 2$ receptor was involved in ECM metabolism, IL-13R $\alpha 2$ gene expression in NP cells was silenced by IL-13R $\alpha 2$-siRNA, and successful IL-13R $\alpha 2$ knockdown was confirmed by western blotting (Figures 5B, C). IL-13R $\alpha 2$ knockdown NP cells were then incubated with rCHI3L1 and western blotting analysis showed that IL-13R $\alpha 2$ receptor knockdown blocked the rCHI3L1-induced ECM metabolic imbalance in NP cells (Figures 5D, E). These results suggest that IL-13R $\alpha 2$ may function as a receptor and is involved in CHI3L1-induced ECM degradation in NP cells.

\section{Mechanism Underlying Contribution of CHI3L1 to ECM Degradation}

To characterize further the mechanism underlying the effects of CHI3L1 on ECM metabolism in NP cells, we investigated MAPK 
A

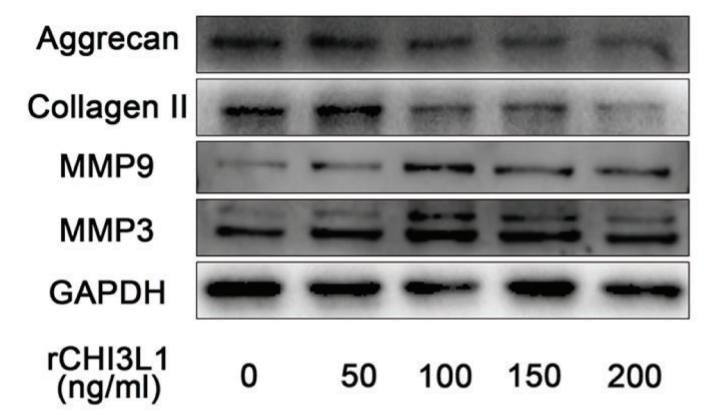

C

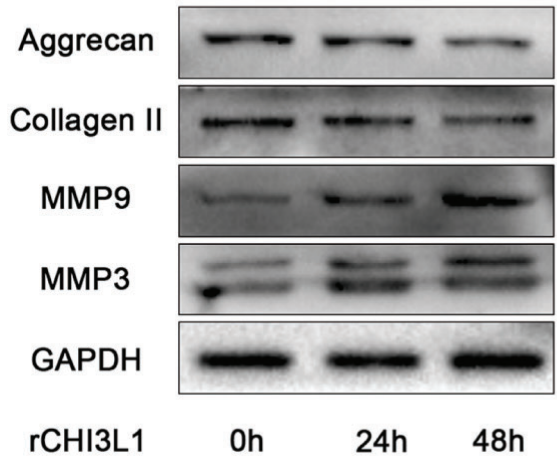

B

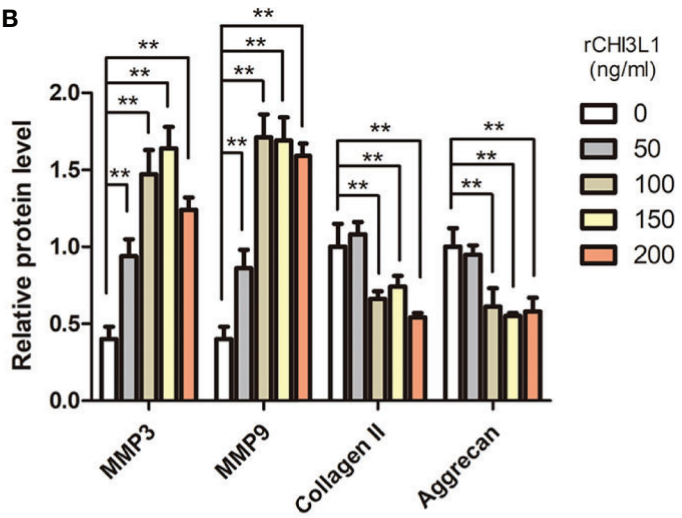

D

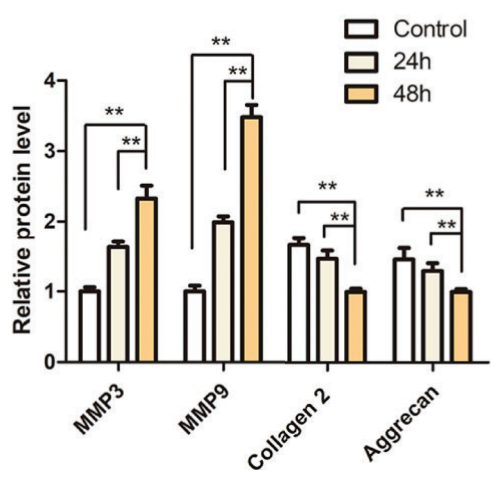

FIGURE 4 | rCHI3L1 promoted ECM metabolism in NP cells. (A) Effects of rCHI3L1 concentration gradient on ECM metabolism in NP cells. Following incubation with different doses of $\mathrm{rCH} \mid 3 \mathrm{~L} 1$ for $48 \mathrm{~h}$, the protein levels of MMP3, MMP9, collagen II and aggrecan in NP cells were detected by western blotting.

(B) Quantification of the protein levels in (A, C) Effects of rCHI3L1 on ECM metabolism at different time points. NP cells were treated with $\mathrm{rCH} 3 \mathrm{~L}$ 1 (150 $\mathrm{ng} / \mathrm{ml}$ ) for 24 or $48 \mathrm{~h}$. The protein levels of MMP3, MMP9, collagen II and aggrecan were detected by western blotting. (D) Quantification of the protein levels in (C) Data are presented as means \pm standard deviations $(n=3)$ from one representative experiment of three independent experiments performed in triplicate. The dates were analyzed by One-way analysis of variance (ANOVA). Significant differences between groups are shown as ${ }^{\star *} \mathrm{p}<0.01$.

pathway activation. Western blotting analysis indicated that rCHI3L1 markedly induced the phosphorylation of ERK and JNK, although there was no substantial change in p38 phosphorylation (Figures 6A, B). Furthermore, western blotting analysis showed that IL-13R $\alpha 2$ receptor blockage suppressed MAPK signaling pathway activation (Figures 6C, D). In addition, exposure to the ERK inhibitor (U0126, $10 \mathrm{nM}$ ) or JNK inhibitor (SP600125, $10 \mathrm{nM}$ ) blocked rCHI3L1-induced ECM metabolic imbalance (Figure 6E). In summary, these results demonstrate that the effects of CHI3L1 on NP cells are specifically mediated by the ERK and JNK pathways, but not the p38 pathway.

\section{DISCUSSION}

In this study, we confirmed the infiltration of M2a macrophages in human and rat degenerated NP tissues. Furthermore, Transwell co-culture experiments demonstrated that M2a macrophages promoted ECM metabolic imbalance in NP cells, at least partly, by secretion of CHI3L1 proteins. Our findings suggested that $\mathrm{CHI} 3 \mathrm{~L} 1$ acted via IL-13R $\alpha 2$ receptors, as well as ERK and JNK signaling pathways. Our results provide insights concerning the effects of M2a macrophages on ECM metabolism in NP cells, as well as potential underlying mechanisms (Figure 7).

Although there has been extensive investigation of macrophage infiltration in disc tissues (31), there has been minimal research regarding specific types of macrophages in this process (32). To our knowledge, this is the first report to explore the effects of M2a macrophages in IDD. Also, we have not found substantial reports concerning the involvement of specific molecules or macrophage components (33). As a macrophage-secreted protein, $\mathrm{CHI} 3 \mathrm{~L} 1$ has been widely researched in various tumors $(17,18)$. Consistent with the findings in studies of tumors, we found that M2a macrophages secreted CHI3L1 proteins, thereby promoting ECM degradation. However, we noted that our findings were different from those of Wang et al. (34), who recently suggested that CHI3L1 may be a protective factor against IDD. In their 
A

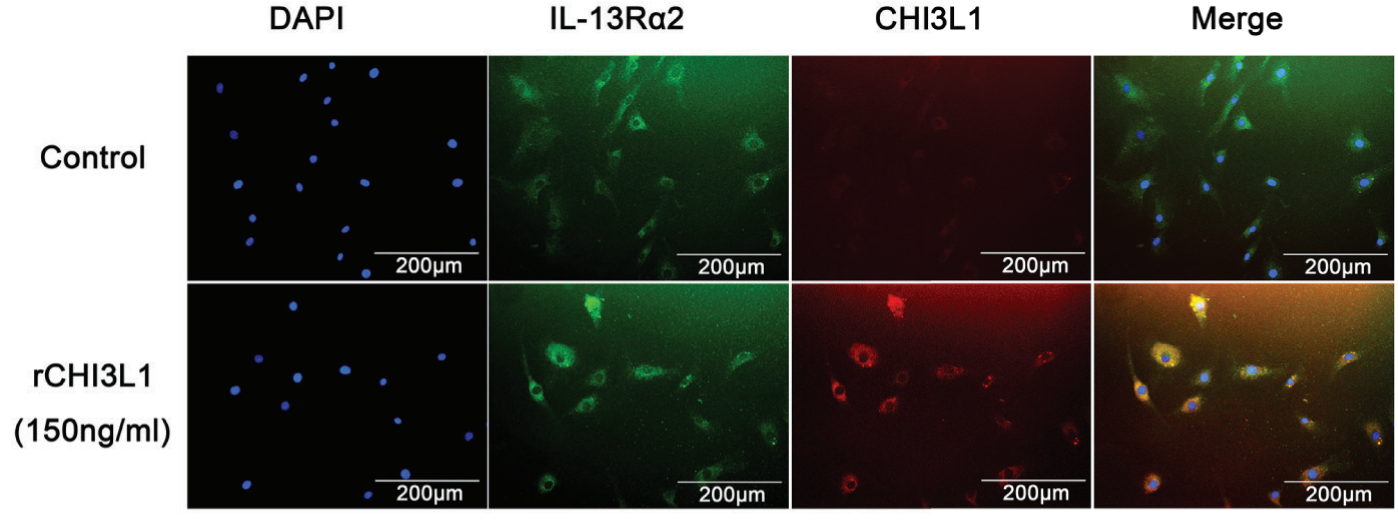

B

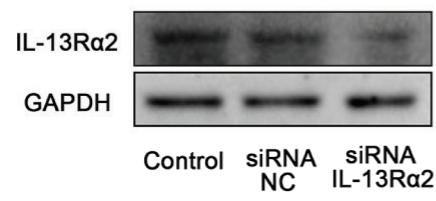

D

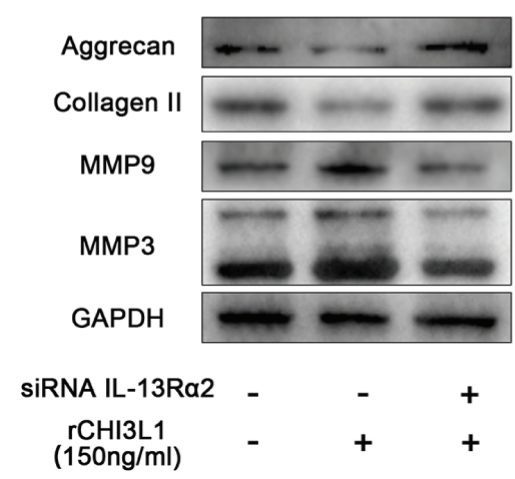

C

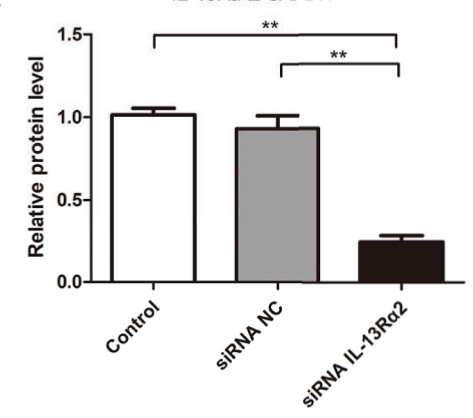

E

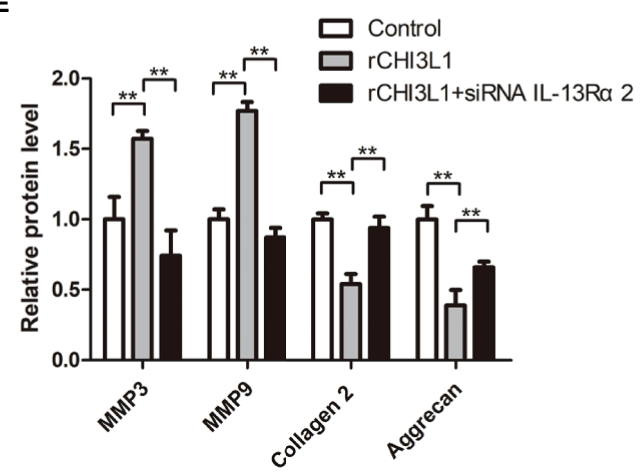

FIGURE 5 | IL-13R $\alpha 2$ was involved in CHI3L1-induced ECM metabolism. (A) Co-localization of CHI3L1 and IL-13R $\alpha 2$ proteins in NP cells. After treatment with rCHI3L1 $(150 \mathrm{ng} / \mathrm{ml})$ for $24 \mathrm{~h}$ double immunofluorescence staining was performed on NP cells with anti-CHI3L1 and anti-IL-13R $\alpha 2$ antibodies. (B) IL-13R $\alpha 2$ was silenced in NP cells by siRNA. NP cells were transfected with NC siRNA or IL-13R $\alpha 2$ siRNA, then grown for $24 \mathrm{~h}$. Knockdown of IL-13R $\alpha 2$ was confirmed by western blotting. (C) Quantification of the protein levels of IL-13R $\alpha 2$ in western blots. (D) Effects of IL-13R $\alpha 2$ silencing on CHI3L1-induced ECM metabolism. After transfecting with NC siRNA or IL-13R 22 siRNA for $24 \mathrm{~h}$, NP cells were incubated with rCHI3L1 (150 ng/ml) for another $24 \mathrm{~h}$. The protein levels of MMP3, MMP9, collagen II, and aggrecan were detected by western blotting. (E) Quantification of the protein levels in (D). Data are presented as means \pm standard deviations $(n=3)$ from one representative experiment of three independent experiments performed in triplicate. The dates were analyzed by One-way analysis of variance (ANOVA). Significant differences between groups are shown as ${ }^{\star *} p<0.01$.

experiments, CHI3L1 overexpression or knockdown was performed in NP cells to explore the role of CHI3L1 expression in those cells. In contrast, we mainly studied M2a macrophage-secreted CHI3L1 protein. This important difference in the source of CHI3L1 may explain the discrepancy between our findings and the results in previous reports.
IL-13R $\alpha 2$ is an important receptor for CHI3L1 and has been extensively studied in the contexts of inflammation, tissue remodeling, and tumors $(35,36)$. This report is the first description of IL-13R $\alpha 2$ involvement in IDD. Furthermore, explorations of downstream MAPK signaling pathways suggested that the ERK and JNK pathways, but not the p38 pathway, mediated these processes. 


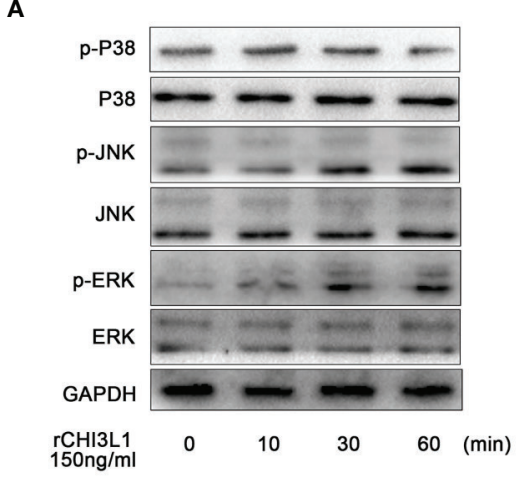

C

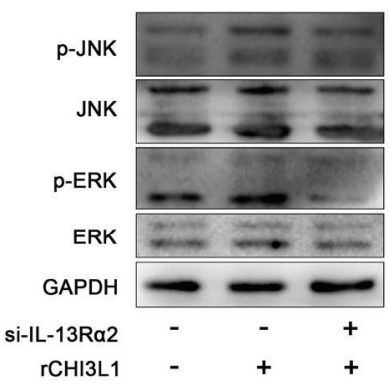

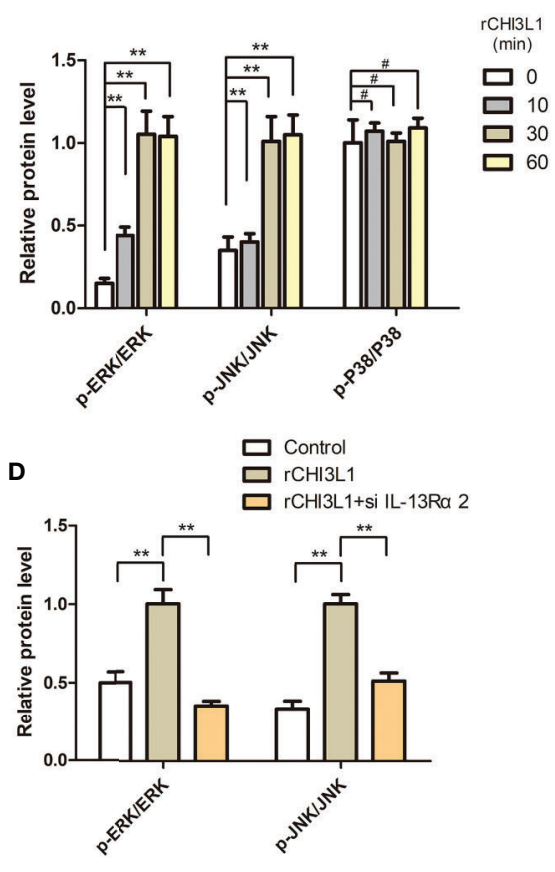

E

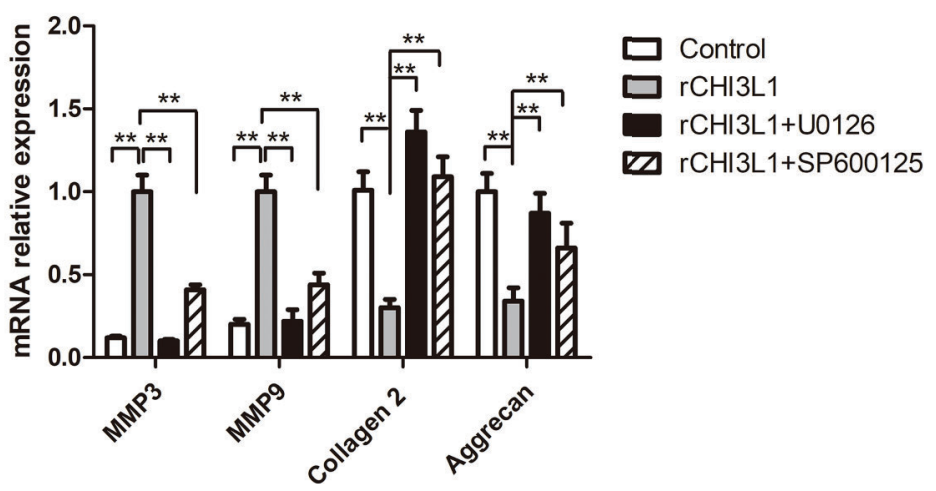

FIGURE 6 | CHI3L1 promoted ECM degradation through the MAPK pathway in NP cells. (A) CHI3L1 affected the MAPK pathway at different time points. NP cells were incubated with rCHI3L1 (150 ng/ml) at different time points (10, 30, and $60 \mathrm{~min})$. The MAPK activation was detected by western blotting. (B) Quantification of the protein levels in $(\mathbf{A}, \mathbf{C}) \mathrm{IL}-13 \mathrm{R} \alpha 2$ was involved in the CHI3L1-induced activation of the MAPK pathway. The IL-13Ro2-silenced NP cells were incubated with rCHI3L1 (150 ng/ml) for $60 \mathrm{~min}$. The MAPK pathway related proteins were detected by western blotting. (D) Quantification of the protein levels in (C). (E) The MAPK signaling pathway was involved in CHI3L1-induced ECM degradation. After treatment with U0126 (ERK inhibitor) or SP600125 (JNK inhibitor) at $10 \mu \mathrm{M}$ for $6 \mathrm{~h}$, NP cells were incubated with rCHI3L1 $(150 \mathrm{ng} / \mathrm{ml})$ for $24 \mathrm{~h}$. MMP3, MMP9, collagen II, and aggrecan expression levels were analyzed by qRT-PCR. Data are presented as means \pm standard deviations $(n=3)$ from one representative experiment of three independent experiments performed in triplicate. The dates were analyzed by One-way analysis of variance (ANOVA). Significant differences between groups are shown as ${ }^{* \star} \mathrm{p}<0.01$. \#, no statistical difference.

There were some limitations in this study. First, it was difficult to obtain completely normal disc tissues from surgery. This study used the most common approach, which involves acquisition of relatively normal disc tissues from patients with adolescent idiopathic scoliosis or congenital scoliosis without known or macroscopically evident disc pathology (22). Second, the macrophages and disc cells from different species, to some extent, may affect the results. On the other hand, CHI3L1 is highly conserved among species, which may partially mitigate the impact. Third, this study did not investigate the expression of macrophage markers by resident NP cells. Notably, Jones et al. found that endogenous disc cells could undergo differentiation to become phagocytes, including the development of phagocytic capacity (37). Here, we identified CD206-positive resident NP 


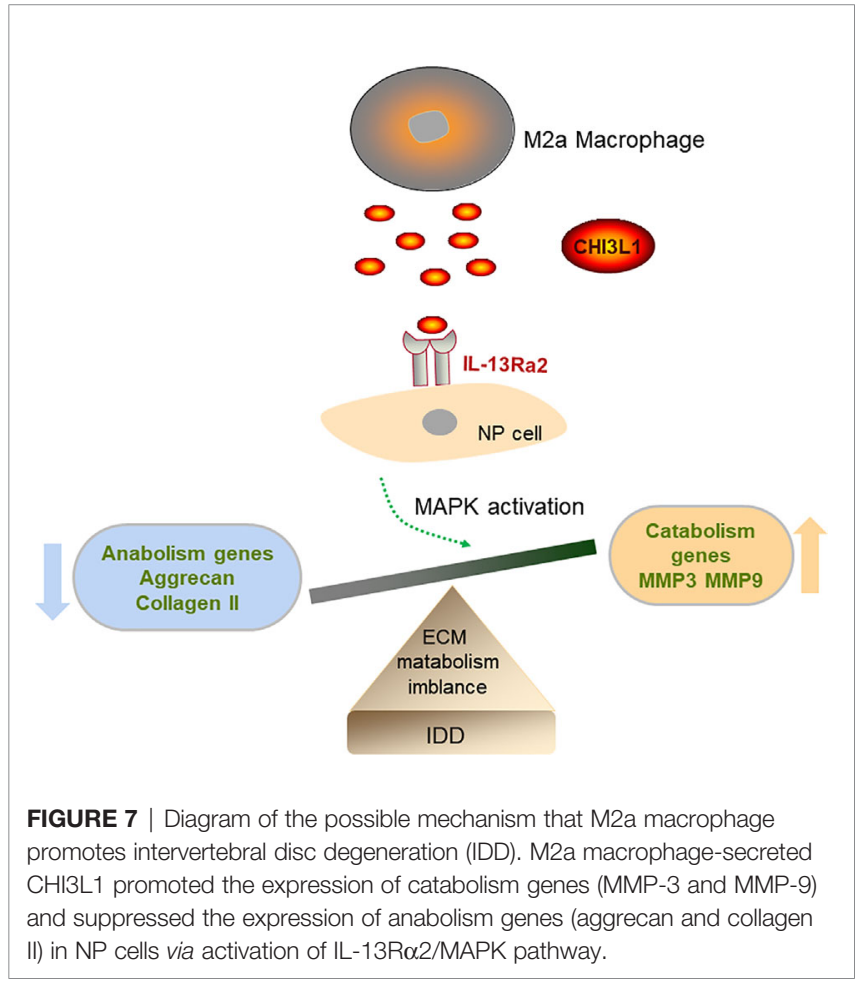

cells in the rat disc degeneration model. Therefore, it may be difficult to distinguish macrophage-like NP cells from true macrophages in intervertebral disc tissues, which requires additional investigation.

In conclusion, this is the first study to explore the roles of M2a macrophages in IDD and the underlying mechanisms of these effects. The results provide additional insights concerning IDD pathophysiology, which may aid in the development of targeted therapeutic strategies.

\section{REFERENCES}

1. Hartvigsen J, Hancock MJ, Kongsted A, Louw Q, Ferreira ML, Genevay S, et al. What Low Back Pain is and Why We Need to Pay Attention. Lancet (2018) 391(10137):2356-67. doi: 10.1016/s0140-6736(18)30480-x

2. Hurwitz EL, Randhawa K, Yu H, Cote P, Haldeman S. The Global Spine Care Initiative: A Summary of the Global Burden of Low Back and Neck Pain Studies. Eur Spine J (2018) 27(Suppl 6):796-801. doi: 10.1007/s00586-0175432-9

3. Cheung KM. The Relationship Between Disc Degeneration, Low Back Pain, and Human Pain Genetics. Spine J (2010) 10(11):958-60. doi: 10.1016/ j.spinee.2010.09.011

4. Ruiz Wills C, Foata B, Gonzalez Ballester MA, Karppinen J, Noailly J. Theoretical Explorations Generate New Hypotheses About the Role of the Cartilage Endplate in Early Intervertebral Disk Degeneration. Front Physiol (2018) 9:1210. doi: 10.3389/fphys.2018.01210

5. Zhang X, Chen J, Huang B, Wang J, Shan Z, Liu J, et al. Obesity Mediates Apoptosis and Extracellular Matrix Metabolic Imbalances Via MAPK Pathway Activation in Intervertebral Disk Degeneration. Front Physiol (2019) 10:1284. doi: 10.3389/fphys.2019.01284

6. Nerlich AG, Weiler C, Zipperer J, Narozny M, Boos N. Immunolocalization of Phagocytic Cells in Normal and Degenerated

\section{DATA AVAILABILITY STATEMENT}

The original contributions presented in the study are included in the article/supplementary materials, further inquiries can be directed to: fanghuangtjh@126.com.

\section{ETHICS STATEMENT}

The studies involving human participants were reviewed and approved by Ethics Committee of Tongji Hospital, Tongji Medical College, Huazhong University of Science and Technology. Written informed consent to participate in this study was provided by the participants' legal guardian/next of kin. The animal study was reviewed and approved by Ethics Committee of Tongji Hospital, Tongji Medical College, Huahong University of Science and Technology.

\section{AUTHOR CONTRIBUTIONS}

LL, KW, and HW conceived the project, designed the experiments and wrote the paper. LL, KW, and YD performed the experiments, analyzed the data. HF reviewed and edited the paper. HX and PA helped to perform the animal experiments and imaging experiments. All authors contributed to the article and approved the submitted version.

\section{FUNDING}

This research was funded by the National Natural Science Foundation of China, grant number 81271347.

Intervertebral Discs. Spine (2002) 27(22):2484-90. doi: 10.1097/ 00007632-200211150-00012

7. Kokubo Y, Uchida K, Kobayashi S, Yayama T, Sato R, Nakajima H, et al. Herniated and Spondylotic Intervertebral Discs of the Human Cervical Spine: Histological and Immunohistological Findings in 500 En Bloc Surgical Samples. Laboratory Investigation. J Neurosurg Spine (2008) 9(3):285-95. doi: $10.3171 /$ spi/2008/9/9/285

8. Ni L, Zheng Y, Gong T, Xiu C, Li K, Saijilafu, et al. Proinflammatory Macrophages Promote Degenerative Phenotypes in Rat Nucleus Pulpous Cells Partly Through ERK and JNK Signaling. J Cell Physiol (2019) 234 (5):5362-71. doi: 10.1002/jcp.27507

9. Yang H, Liu B, Liu Y, He D, Xing Y, An Y, et al. Secreted Factors From Intervertebral Disc Cells and Infiltrating Macrophages Promote Degenerated Intervertebral Disc Catabolism. Spine (2019) 44(9):E520-9. doi: 10.1097/ brs.0000000000002953

10. Byvaltsev VA, Kolesnikov SI, Bardonova LA, Belykh EG, Korytov LI, Giers $\mathrm{MB}$, et al. Development of an In Vitro Model of Inflammatory Cytokine Influences on Intervertebral Disk Cells in 3D Cell Culture Using Activated Macrophage-Like Thp-1 Cells. Bull Exp Biol Med (2018) 166(1):151-4. doi: 10.1007/s10517-018-4304-6

11. Mosser DM, Edwards JP. Exploring the Full Spectrum of Macrophage Activation. Nat Rev Immunol (2008) 8(12):958-69. doi: 10.1038/nri2448 
12. Murray PJ, Allen JE, Biswas SK, Fisher EA, Gilroy DW, Goerdt S, et al. Macrophage Activation and Polarization: Nomenclature and Experimental Guidelines. Immunity (2014) 41(1):14-20. doi: 10.1016/j.immuni.2014.06.008

13. Spiller KL, Anfang RR, Spiller KJ, Ng J, Nakazawa KR, Daulton JW, et al. The Role of Macrophage Phenotype in Vascularization of Tissue Engineering Scaffolds. Biomaterials (2014) 35(15):4477-88. doi: 10.1016/j.biomaterials.2014.02.012

14. Nakazawa KR, Walter BA, Laudier DM, Krishnamoorthy D, Mosley GE, Spiller KL, et al. Accumulation and Localization of Macrophage Phenotypes With Human Intervertebral Disc Degeneration. Spine J (2018) 18(2):343-56. doi: 10.1016/j.spinee.2017.09.018

15. Latta CH, Sudduth TL, Weekman EM, Brothers HM, Abner EL, Popa GJ, et al. Determining the Role of IL-4 Induced Neuroinflammation in Microglial Activity and Amyloid-Beta Using BV2 Microglial Cells and APP/PS1 Transgenic Mice. J Neuroinflamm (2015) 12:41. doi: 10.1186/s12974-0150243-6

16. Yeo IJ, Lee CK, Han SB, Yun J, Hong JT. Roles of Chitinase 3-Like 1 in the Development of Cancer, Neurodegenerative Diseases, and Inflammatory Diseases. Pharmacol Ther (2019) 203:107394. doi: 10.1016/j.pharmthera.2019.107394

17. Qiu QC, Wang L, Jin SS, Liu GF, Liu J, Ma L, et al. CHI3L1 Promotes Tumor Progression by Activating TGF-Beta Signaling Pathway in Hepatocellular Carcinoma. Sci Rep (2018) 8(1):15029. doi: 10.1038/s41598-018-33239-8

18. Chen Y, Zhang S, Wang Q, Zhang X. Tumor-Recruited M2 Macrophages Promote Gastric and Breast Cancer Metastasis Via M2 Macrophage-Secreted CHI3L1 Protein. J Hematol Oncol (2017) 10(1):36. doi: 10.1186/s13045-0170408-0

19. Di Rosa M, Szychlinska MA, Tibullo D, Malaguarnera L, Musumeci G. Expression of CHI3L1 and CHIT1 in Osteoarthritic Rat Cartilage Model. A Morphological Study. Eur J Histochem (2014) 58(3):2423. doi: 10.4081/ejh.2014.2423

20. Kumagai E, Mano Y, Yoshio S, Shoji H, Sugiyama M, Korenaga M, et al. Serum YKL-40 as a Marker of Liver Fibrosis in Patients With non-Alcoholic Fatty Liver Disease. Sci Rep (2016) 6:35282. doi: 10.1038/srep35282

21. Gordon S, Martinez FO. Alternative Activation of Macrophages: Mechanism and Functions. Immunity (2010) 32(5):593-604. doi: 10.1016/j.immuni.2010.05.007

22. Teixeira GQ, Yong Z, Goncalves RM, Kuhn A, Riegger J, Brisby H, et al. Terminal Complement Complex Formation is Associated With Intervertebral Disc Degeneration. Eur Spine J (2021) 30(1):217-26. doi: 10.1007/s00586-02006592-4

23. Ge J, Yan Q, Wang Y, Cheng X, Song D, Wu C, et al. IL-10 Delays the Degeneration of Intervertebral Discs by Suppressing the P38 MAPK Signaling Pathway. Free Radical Biol Med (2020) 147:262-70. doi: 10.1016/ j.freeradbiomed.2019.12.040

24. Peng X, Zhang C, Bao JP, Zhu L, Shi R, Xie ZY, et al. A20 of Nucleus Pulposus Cells Plays a Self-Protection Role Via the Nuclear Factor-Kappa B Pathway in the Inflammatory Microenvironment. Bone Joint Res (2020) 9(5):225-35. doi: 10.1302/2046-3758.95.bjr-2019-0230.r1

25. Wang Y, Zuo R, Wang Z, Luo L, Wu J, Zhang C, et al. Kinsenoside Ameliorates Intervertebral Disc Degeneration Through the Activation of AKT-ERK1/2-Nrf2 Signaling Pathway. Aging (2019) 11(18):7961-77. doi: 10.18632/aging.102302

26. Spencer M, Finlin BS, Unal R, Zhu B, Morris AJ, Shipp LR, et al. Omega-3 Fatty Acids Reduce Adipose Tissue Macrophages in Human Subjects With Insulin Resistance. Diabetes (2013) 62(5):1709-17. doi: 10.2337/db12-1042

27. Raghavan S, Mehta P, Xie Y, Lei YL, Mehta G. Ovarian Cancer Stem Cells and Macrophages Reciprocally Interact Through the WNT Pathway to Promote
Pro-Tumoral and Malignant Phenotypes in 3D Engineered Microenvironments. J Immunother Cancer (2019) 7(1):190. doi: 10.1186/ s40425-019-0666-1

28. Liao Z, Luo R, Li G, Song Y, Zhan S, Zhao K, et al. Exosomes From Mesenchymal Stem Cells Modulate Endoplasmic Reticulum Stress to Protect Against Nucleus Pulposus Cell Death and Ameliorate Intervertebral Disc Degeneration In Vivo. Theranostics (2019) 9(14):4084-100. doi: 10.7150/ thno. 33638

29. Lee SH, Lee S, Du J, Jain K, Ding M, Kadado AJ, et al. Mitochondrial MsrB2 Serves as a Switch and Transducer for Mitophagy. EMBO Mol Med (2019) 11 (8):e10409. doi: 10.15252/emmm.201910409

30. Troidl C, Möllmann H, Nef H, Masseli F, Voss S, Szardien S, et al. Classically and Alternatively Activated Macrophages Contribute to Tissue Remodelling After Myocardial Infarction. J Cell Mol Med (2009) 13(9b):3485-96. doi: 10.1111/j.1582-4934.2009.00707.x

31. Lee S, Millecamps M, Foster DZ, Stone LS. Long-Term Histological Analysis of Innervation \& Macrophage Infiltration in a Mouse Model of Intervertebral Disc Injury-Induced Low Back Pain. J Orthop Res (2020) 38(6):1238-47. doi: $10.1002 /$ jor. 24560

32. Byvaltsev VA, Kolesnikov SI, Bardonova LA, Belykh EG, Korytov LI, Giers MB, et al. Assessment of Lactate Production and Proteoglycans Synthesis by the Intact and Degenerated Intervertebral Disc Cells Under the Influence of Activated Macrophages: An In Vitro Study. Bull Exp Biol Med (2018) 166(1):170-3. doi: 10.1007/s10517-0184307-3

33. Silva AJ, Ferreira JR, Cunha C, Corte-Real JV, Bessa-Goncalves M, Barbosa MA, et al. Macrophages Down-Regulate Gene Expression of Intervertebral Disc Degenerative Markers Under a Pro-inflammatory Microenvironment. Front Immunol (2019) 10:1508. doi: 10.3389/fimmu.2019.01508

34. Wang R, Xu C, Zhong H, Hu B, Wei L, Liu N, et al. Inflammatory-Sensitive CHI3L1 Protects Nucleus Pulposus Via AKT3 Signaling During Intervertebral Disc Degeneration. FASEB J (2020) 34(3):3554-69. doi: 10.1096/ fj.201902096R

35. He CH, Lee CG, Dela Cruz CS, Lee CM, Zhou Y, Ahangari F, et al. Chitinase 3-Like 1 Regulates Cellular and Tissue Responses Via IL-13 Receptor Alpha2. Cell Rep (2013) 4(4):830-41. doi: 10.1016/j.celrep.2013.07.032

36. He CH, Lee CG, Ma B, Kamle S, Choi AMK, Elias JA. N-Glycosylation Regulates Chitinase 3-Like-1 and IL-13 Ligand Binding to IL-13 Receptor Alpha2. Am J Respir Cell Mol Biol (2020) 63(3):386-95. doi: 10.1165/ rcmb.2019-0446OC

37. Jones P, Gardner L, Menage J, Williams GT, Roberts S. Intervertebral Disc Cells as Competent Phagocytes In Vitro: Implications for Cell Death in Disc Degeneration. Arthritis Res Ther (2008) 10(4):R86. doi: 10.1186/ar2466

Conflict of Interest: The authors declare that the research was conducted in the absence of any commercial or financial relationships that could be construed as a potential conflict of interest.

Copyright (c) $2021 \mathrm{Li}$, Wei, Ding, Ahati, Xu, Fang and Wang. This is an open-access article distributed under the terms of the Creative Commons Attribution License (CC BY). The use, distribution or reproduction in other forums is permitted, provided the original author(s) and the copyright owner(s) are credited and that the original publication in this journal is cited, in accordance with accepted academic practice. No use, distribution or reproduction is permitted which does not comply with these terms. 